\title{
Complex Abdominal Wall Defects and Enterocutaneous Fistulae in the Era of Biological Mesh: Did We Make any Real Progress?
}

\author{
Rifat Latifi • Ari Leppäniemi
}

Published online: 1 October 2011

(C) Société Internationale de Chirurgie 2011

Significant advances in surgical techniques and technologies have occurred in the last few decades. New developments in highly sophisticated trauma and critical care, specialized nutritional support, nursing care, pharmaceutical advances, as well as advanced knowledge of biology and anatomy of injuries, have considerably advanced surgical care in the twenty-first century. Mortality and morbidity of trauma and emergency surgery has never been lower in the history of mankind.

The concept of damage control surgery, or abbreviated laparotomy and planned reoperation, has been accepted as a new paradigm to treat the most severely injured and physiologically compromised patients, and it has been increasingly used in patients with non-traumatic abdominal emergencies, such as severe acute pancreatitis or secondary peritonitis. Also, the recognition of increased intraabdominal pressure, and especially abdominal compartment syndrome, as a condition requiring active monitoring and sometimes surgical decompression has increased the number of patients with abdomens left open with a temporary cover of the viscera. At the same time, we have made significant progress, both in technology and in knowledge and

R. Latifi ( $\square)$

University of Arizona, 1501 Campbell Avenue,

Tucson, AZ 85749, USA

e-mail: latifi@surgery.arizona.edu

R. Latifi

Trauma Services, Hamad Medical Corporation,

P.O. Box 3050, Doha, Qatar

\author{
A. Leppäniemi \\ Department of Abdominal Surgery, Meilahti Hospital, \\ University of Helsinki, Haartmaninkatu 4, \\ P.O. Box 340, 00029 Helsinki, Finland \\ e-mail: ari.leppaniemi@hus.fi
}

skills, in managing patients with open abdomens. Nevertheless, open abdomen, or laparostomy, is still a morbid procedure with potentially severe consequences. The end result is often a patient who has survived the initial insult, has an open abdomen with temporary cover requiring delayed reconstruction of the abdominal wall defect or giant ventral hernia, or, in the worst case, a frozen abdomen with enteric fistulas. Preventing and learning to manage these complications is of utmost importance to justify the use of this life-saving intervention.

Furthermore, the field of elective surgery is changing. Gone are the days of gastric resections for ulcer diseases. Our population is getting more obese, and we have invented a new operation for this new disease called bariatric surgery, open or laparoscopic. In spite of advances made in surgical techniques and perioperative care, bariatric surgery can be associated with severe complications, including anastomotic leaks, wound infections, and sometimes with a giant abdominal wall defect, complex in itself, and even more so in a morbidly obese patient.

To this end, surgeons are faced with an almost new entity: major abdominal wall defects in young and old patients with multiple co-morbid diseases. Surgical repair of these hernias and defects is complex, despite all of the advances that that have been made in surgery. The recurrence rates of repaired abdominal wall defects and complex hernias, especially ventral hernias, remain quite high among surgical patients. It is not clear how many of these are classified as "complex," but it is clear that ventral hernias and other abdominal wall defects represent a significant surgical problem with great morbidity and mortality. Incisional hernias are large and over time can result in serious complications such as pain, bowel obstruction, incarceration and strangulation, and enterocutaneous fistulae. Furthermore, the quality of life and opportunities for 
employment are reduced in patients suffering from incisional hernias. Patients get into a vicious cycle. The lack of abdominal wall and or large hernia protruding through the abdominal wall into the subcutaneous tissue inhibits patients' ability to work, walk, or exercise. This leads to further weight gain, exacerbating the already complex pathology and making it worse for the patients. A few surgeons refuse to operate altogether, or insist that the patient lose weight before they can be considered an "acceptable surgical candidate"! Although such an approach perhaps makes sense, we all know that in practice, we rarely see a patient who truly succeeds in shedding kilograms in order to have surgery. Thus, repair of such hernias is truly complicated by many factors, including psychosocial, economic, protein malnutrition, and hygiene, among others.

The IATSIC symposium proceedings presented in this issue of World Journal of Surgery, illustrate best the complexity of the "new surgical disease." The objective of this symposium was to review the status of the biology of large, complex hernias, with or without enterocutaneous fistulae, and associated conditions such as stomas and surgical techniques applied in their management, especially in the era of biological mesh.

Hernias may arise from prior abdominal surgery and pose a significant challenge for general, trauma, and acute care surgeons; plastic surgeons; urologists; vascular surgeons; and, occasionally, thoracic surgeons. The indications for repair of ventral hernias have been established, but the technique of repair and what type of reinforcement materials should be used remain controversial. Significant numbers of defects in the abdominal wall present complex management problems and are associated with partial or complete loss of the abdominal wall domain, enterocutaneous or colonic fistulae, skin grafts, skin erosions, chronic wounds, and sinus tracts that drain continuously. Bad odor, poor hygiene, and other factors are other issues. These conditions represent significant morbidity and are often the cause of death after the recurrence of sepsis from central lines or total parenteral nutrition, or from recurrent intraabdominal sepsis, urinary sepsis, or another infection arising from the primary injury that caused the fistulae and the loss of abdominal wall domain. The cost of this new disease is not known; however, what is known is that there is an entirely new industry flourishing on the surgical management of these conditions. In particular, the industry promotes the use of new meshes and prostheses, each claiming that it is better than the others. However, persistent seromas, fistulas, and intestinal obstructions from adhesions continue to plague hernia repair and abdominal wall reconstructions.

Most recently, biologic meshes have been introduced into hernia repair surgery as a significant development in the armamentarium of surgeons caring for this group of patients. These patients have significant co-morbid factors, and biologic meshes are nearly the only alternative, especially when concerns for wound infections are present. The ability of certain biologic prostheses to support revascularization and become part of human tissue is a major advance and the chief benefit of these meshes. This has certainly added a new dimension to surgical repair of major abdominal wall defects. The application of combined advanced surgical techniques and biological materials may reduce the risk of recurrence and surgical site complications, such as infection. The biologic mesh that is both human and porcine in origin is useful especially in high risk patients. As demonstrated by studies cited by Latifi et al., the acellular dermal matrix (ADM) provides an advantage over non-biological materials that have been used as an adjunct to hernia repair with allowance of implantation in infected fields. There is a concern, however, that because this is a new field of surgery, no standardized method of repair exists for use of acellular dermal matrix in abdominal wall reconstructions, despite its daily use by a number of surgeons worldwide.

The symposium of IATSIC for the World Journal of Surgery brought together some top notch experts from around the world to address this complex issue. Coimbra and co-workers start off with surgical strategies in the management of open abdomen in trauma and emergency surgery, followed by planned hernia repair and abdominal wall reconstruction by Leppäniemi and Tukiainen. Latifi and colleagues address the reoperative surgical approach in patients with enterocutaneous fistula and describe the techniques of reconstructions using biological mesh, as well as the challenges that surgeons face with these cases. Nutritional management of patients with enterocutaneous fistula (ECF) is covered by Schwab and colleagues by illustrating some of the complex management issues when caring for these patients. Peralta and Latifi analyze the long-term data, and. Abiad and Hoballa takes on management of hernia and abdominal defects in morbidly obese patients.

Overall, we hope that this symposium answers the questions facing all surgeons caring for patients with this complex malady, and that the articles published here shed light on the new and current management of patients with ECF and abdominal defects in the era of biologic mesh. 